\title{
Using Three Op Amp Simple AM/FM Radio Circuit
}

\author{
Min Jiang \\ Ningbo Polytechnic, Ningbo, China \\ Email: chiangm@163.com
}

How to cite this paper: Jiang, M. (2018) Using Three Op Amp Simple AM/FM Radio Circuit. Open Access Library Journal, 5: e4334.

https://doi.org/10.4236/oalib.1104334

Received: January 12, 2018

Accepted: February 10, 2018

Published: February 13, 2018

Copyright (๑) 2018 by author and Open Access Library Inc.

This work is licensed under the Creative Commons Attribution International License (CC BY 4.0).

http://creativecommons.org/licenses/by/4.0/

\section{(c) (†) Open Access}

\begin{abstract}
After nearly a year of probation, the type of AM radio repeater circuit in general can be retrieved in a number of local radio stations; the installation is convenient, the output has a loud voice, and bass is rich, which make it indeed a good choice for beginners' homemade radio circuit. Beginners can give radio principle about double harvest theory and practice. The whole circuit fabrication costs low. The AM circuit is successful, plus USB, TF playing blocks, FM module circuit without external antenna, and the antenna is surrounded by a large box inside a circle around the wire. The two buttons use to control move, and changing frequencies is very convenient. We usually put the electromagnetic spider web test on the radio and put it all over the country.
\end{abstract}

\section{Subject Areas}

Electric Engineering

\section{Keywords}

AM, FM, Direct Radio, LM386 High Release, TDA2822 Power Amplifier

\section{A Brief Description of the Circuit Characteristics}

A circuit diagram of a medium wave radio can also be received in the basement. This radio sensitivity: can clearly and stably receive local broadcasts or strong signal, radio programs, be connected to an external antenna, easily capture all kinds of broadcasting, and increase the voice through the knob. The far sounds come in a throng! It can receive no signal in the basement of the ordinary radio, listening to the medium wave and the electromagnetic interference sound is very few. I tried to test $939 \mathrm{KHz}$, Ningbo traffic radio. The general machine here is not well heard. With the high grade radio received, except the obvious electromagnetic interference sound, it is also accompanied by a large background noise. 
This product in addition to electromagnetic interference sound relatively small and talking about the volume is high crystal clear horn sound as clear azure sky bright moon. The entire channel transparent and boundless is very clear but the original bass heavy speech intelligibility is less; the wave sensitivity is satisfactory. The sound characteristic is: the whole characteristic is the magnificent atmosphere and the thick and balanced. To say the atmosphere, the circuit power supply has chosen the $3.7 \mathrm{~V}$ rechargeable power supply, the $3 \mathrm{~W}$ horn is large and loud, and the whole room is shaking. The sound of the radio that it designs is powerful, not the calm of the brook, but lively, dynamic and strong. Compared with the upscale HIVI sound box, although the sense of hierarchy is lacking and the tone is slightly dark, it is excellent in comparison to the sound I know on the radio. It is more distinctive than any other radio. The sound of the horn that was dismantled in the old machine should be quite outstanding and sound more masculine. Bass is elegant extremely and valuable. Voice is very soft and slightly divergent, flat and thin; only discrimination can feel that it is not the general. It is strange for the first time to be installed, and the interference is eliminated only with the length of the rubber multipoint connection. After installing the circuit in the first step, $5 \sim 8$ radio stations can normally be received [1] [2].

\section{LC Tuning Circuit}

The diameter of $10 \mathrm{~mm}$, length $140 \mathrm{~mm}$ (also available $160 \mathrm{~mm}$ ) MnZn MXO400 material polish bar type Mn: initial permeability of MnZn ferrite 400, it is a black, low frequency and high permeability, proved by experiments with multi strand coil than by single strand wire winding, there are more obvious increase in sensitivity and selectivity. Here the yarn such as enameled wire with a diameter of $0.05 \mathrm{~mm} 13$ root twisted into a wound after (usually used $7 \times 0.07 \mathrm{~mm}$ or $15 \times 0.05 \mathrm{~mm}$ enameled wire). The number of shares increased much, and the performance increased little. If the $\mathrm{Q}$ value was less, the value would be reduced. It is suitable for the medium wave L1 to use multi strand yarn enameled wire L1 (90 turn) and L2 (10 turn) distance $10 \mathrm{~mm}$. The effect is satisfactory, and the sensitivity and selectivity are good. If it is possible to remove a magnetic antenna from a waste radio, the use of a plastic coil tube helps to improve the $\mathrm{Q}$ value of the LC tuning loop. The LC loop is generally calculated by the following formula.

$$
f=\frac{1}{2 \pi \sqrt{\mathrm{LC}}}
$$

\section{TA7642 Tuning Circuit}

The circuit of the radio is mainly composed of tuning circuit, high frequency amplifying circuit, detecting circuit, audio amplifying circuit and power amplifying circuit. Through the above circuit, we can amplify and amplify the signal, so that we can hear the content in the radio. The high frequency signal receiving 
in many radio stations from a bar antenna, through the input tuning circuit, so that only the other can we need to successfully enter the TA7642; the high frequency signal transmission over from the input circuit is extremely weak, also must be sufficient amplification, this is referred to as the high frequency amplifier, put. The high frequency signal is detected in the next step. The purpose of the detection is to take down the low frequency signal modulated on the high frequency carrier. The frequency amplification is carried out through the audio amplification circuit, and then the power amplifier is carried out to make the whole machine output audio signal with good sound quality.

For example, when using the $270 \mathrm{pF} \mathrm{C}$ variable capacitor, inductor coil antenna matching is about $250 \mathrm{~Hz}$, the low-end loop coverage frequency is about $525 \mathrm{KHz}$; the minimum capacitance hypothesis of variable capacitor for $20 \mathrm{pF}$ high-end $2200 \mathrm{~Hz}$, so its coverage is $525-2200 \mathrm{KHz}$, can satisfy the receiving medium wave radio frequency $530-1700 \mathrm{KHz}$. If you use a variable capacitance variable capacitance, it is necessary to use this formula to calculate the inductance of the required antenna coil to meet the reception. L1 and L2 in the position of the magnet to note that the L center distance of 1 high; L2 to try to put near the bar at one end, that L2 itself receiving electromagnetic waves will be very weak, strong interference can suppress local wave radio signal. Figures 1-3 is a schematic diagram of a direct radio circuit.

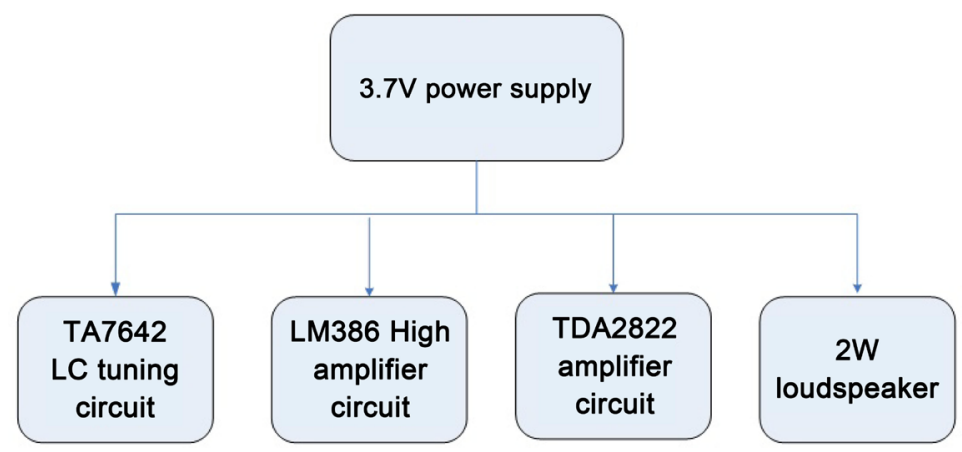

Figure 1. The schematic diagram of the AM radio.

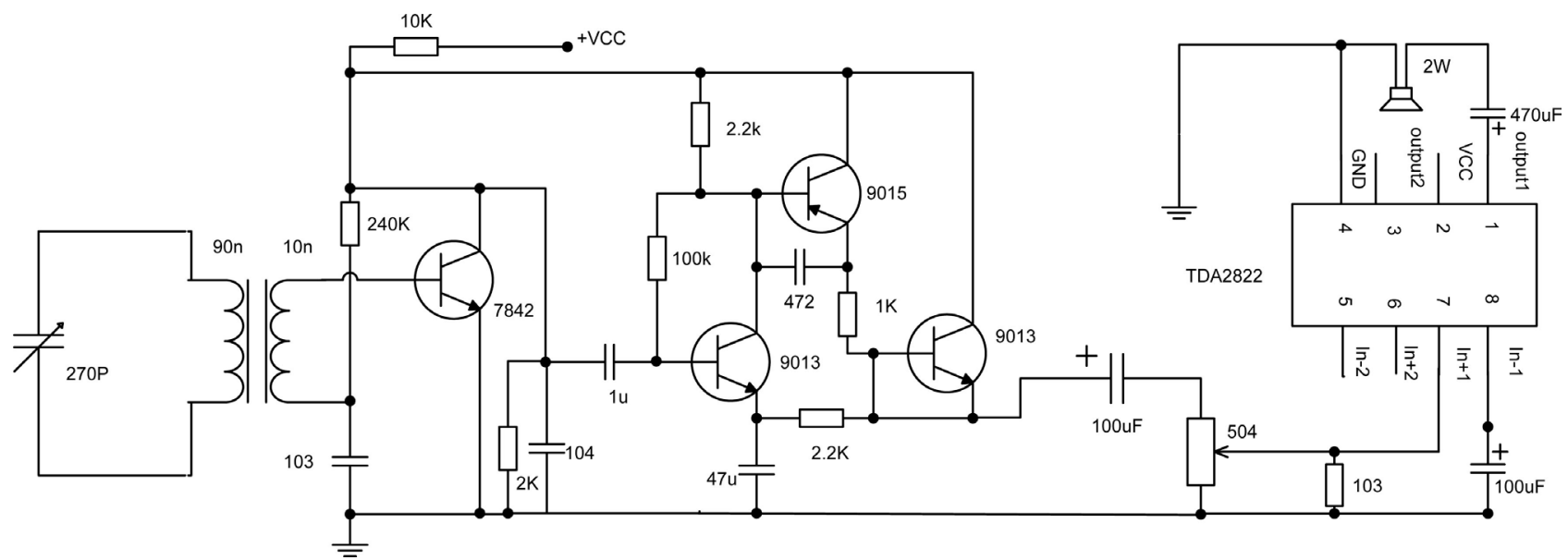

Figure 2. The part of the AM radio 1. 


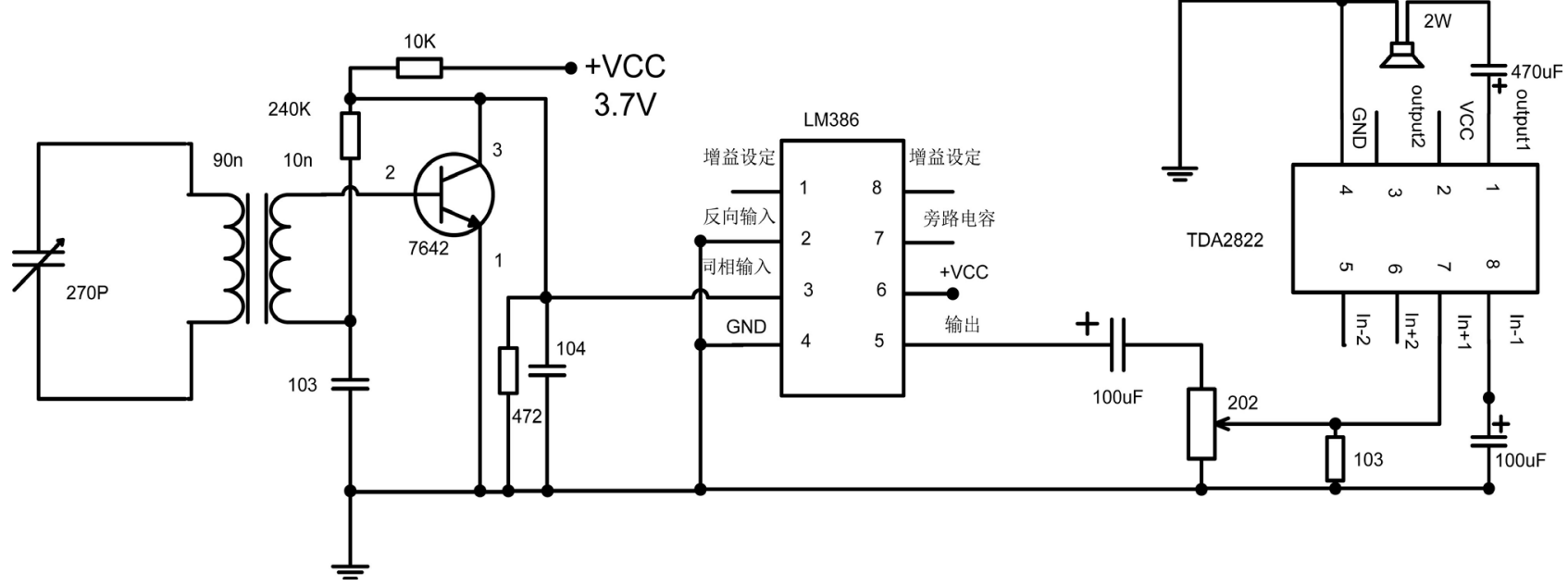

Figure 3. The part of the AM radio 2.

\section{LM386 High Amplifier Circuit}

LM386 is an audio power amplifier produced by the National Semiconductor Corporation Ns. It is mainly used in the high level part of the homemade AM radio. The circumference element is least, and the voltage gain is built in 20 . With an external resistance and capacitance, the voltage gain can be adjusted to a greater extent, and the maximum can be obtained Max to 200. The input end is referenced by status, and the output end is automatically offset to the power supply. Half of the pressure, under the $6 \mathrm{~V}$ power supply, its static power is only $24 \mathrm{~mW}$. LM386 is especially suitable for battery powered occasions, the form of LM386. There are plastic sealing 8 lead double row and patch type. LM386 has low static power consumption, which is about $4 \mathrm{~mA}$, which can be used for battery power supply electricity. The pressure range is wide, $4-12 \mathrm{~V}$ or $5-18 \mathrm{~V}$. There are few peripheral components. The voltage gain is adjustable, $20-200$. Low distortion can be used for direct amplification of a special set of radios a circuit consisting of an 10 only triode, 7 resistors, and several capacitors. The functions include the first level high impedance differential input buffer, the three stage high frequency amplification and the first stage. Main features: high sensitivity, if not the back circuit will be able to use a common headset to hear a clear radio sound; working frequency. Automatic gain control range up to $20 \mathrm{~dB}$ and bandwidth in $250 \mathrm{KHz}-2500 \mathrm{KHz}$ power gain up to $60 \mathrm{~dB}$. Because of the following three reasons, the circuit should be considered to correct:

1) The noise is big and the signal to noise ratio is not good, so the weak radio station is often drowned in a piece of noise. LM386 is an audio integrated power amplifier. It has many advantages, such as low power consumption, 1, 8 terminal gain adjustment, high power supply voltage range, small external components and total harmonic distortion.

2) The gain is insufficient. The loud and loud voice can be heard when connected to the high impedance earphone, but the quality of the chip is not enough. Even with TDA2822 and other power amplifiers, the noise is small and 
the sensitivity is not enough. Not SANGEAN radio as good sound quality, so it must be changed to LM386.

3) TA7642 is a micro integrated circuit with the same shape of the triode. Its interior is integrated with 10 tri-transistor, including three-stage high-level, first-stage and first-stage low frequency buffer amplification. But the input impedance is not high enough. After LC selective tuning circuit basically enough, but the poor quality of the input impedance of the chip is not high enough, if direct access to the LC tuning circuit significantly affect the selectivity, while in LM386 it can be used with around the secondary coil in the magnetic antenna can be solved. It is necessary to do second. At the installation of five sets using the 2018 New Year's Day, the AM circuit is a successful, plus USB, TF playing blocks, FM module circuit without external antenna, the antenna is surrounded by a large box inside a circle around the wire, the two buttons to move, change is very convenient. This product proves simple and practical is the hard truth; it is suitable for the convenience of beginners to test.

\section{TDA2822 Power Amplifier Circuit}

If the horn output is to be driven, it is necessary to connect the TDA2822 audio power amplifier circuit. The following characteristics: the circuit supply voltage range $(1.8-15 \mathrm{~V})$, the power supply voltage can be as low as $1.8 \mathrm{~V}$ can still work, therefore, the circuit is suitable for working under low voltage supply; small quiescent current, the crossover distortion is small; suitable for mono bridge (BTL) or stereo line two working state by 8 feet; dual in-line plastic package (DIP8). The homemade AM direct radio circuit is only one way to enlarge the circuit, and the 5 is the PCB diagram of the direct radio, which is completed with the ARES in Proteus.

Figure 4 is using three Op Amp simple AM/FM radio.

\section{Summary}

The purpose of the design of the radio is to have the practical use of the electromagnetic spider web equipment in many places. After nearly a year of probation,

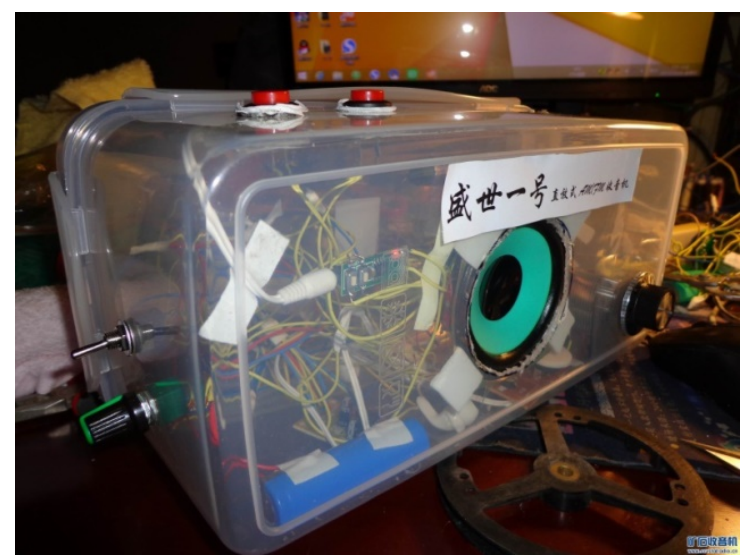

Figure 4. Using three Op Amp simple AM/FM radio circuit. 
the type of AM, FM radio repeater circuit in general can be retrieved in a number of local radio stations; the installation is convenient, the output has a loud voice, and rich bass is indeed a good choice for beginners' homemade radio circuit. Beginners can give radio principle about double harvest theory and practice. The whole circuit fabrication costs less than 50 Yuan. We usually put the electromagnetic spider web test on the radio and put it all over the country. The electromagnetic spider web node is often taken care of.

\section{References}

[1] Jiang, M. (2010) Earthquake Prediction Micro Integrated Measurement Recorder. Technology Innovation Herald, No. 29, 22-23.

[2] Nguyen, C.T.-C. (1999) Frequency-Selective MEMS for Miniaturized Low-Power Communication Devices. IEEE Transactions on Microwave Theory and Techniques, 47, 1486-1489. https://doi.org/10.1109/22.780400 